\title{
Modelo de análisis y formulación estratégica. Empleando herramientas matriciales
}

\section{RESUMEN}

El análisis y formulación estratégica es un proceso de evaluación y preparación del diseño del futuro de un sector, un negocio o una empresa. El objetivo es elaborar los elementos para la discusión y unificación de las diversas concepciones directivas. Las fases del análisis estratégico son cinco: 1) Fase de entrada, resume la data inicial y plantea la posición estratégica de empresa y productos; 2) Fase de análisis, integra factores externos e internos y plantea las alternativas estratégicas; 3) Fase de salida, consolida las estrategias considerando su factibilidad técnica; 4) Fase de calificación de las alternativas que cumplen la condición de factibilidad y deseabilidad; 5) Evaluación y decisión, eligiendo estrategias que generan valor a la empresa.

El resultado esperado considera que, de una situación inicial donde el análisis y la proyección de largo plazo son insuficientes o inexistentes, se llega a una nueva situación donde se poseen las herramientas adecuadas para la preparación y elección de estrategias factibles y deseables.

Palabras clave: Análisis estratégico, formulación estratégica, decisión estratégica.

Model OF STRATEgiC ANALYSIS AND FORMULATION. USING MATRIX TOOLS

\section{ABSTRACT}

The analysis and strategic formulation is a strategic assessment and preparation process of designing the future of industry, business or enterprise. The goal is to develop some elements for discussion and unification of several directives conceptio$n s$. The phases of strategic analysis are five: 1) Input phase, summarizes initial data and raises the company's strategic position and products, 2) Analysis phase, integrates external and internal factors and raises strategic alternatives, 3) Output phase consolidate all strategies considering their technical feasibility, 4) Qualifying phase of the alternatives that fulfill the condition $F+D$ and create value, 5) Evaluation and decision, choosing strategies that deliver value to the company. The expected result considers that, from an initial situation where analysis and long-term projection of insufficient or non-existent it passes to new situation where it has right tools for the preparation and choice of strategies feasible and desirable.

KEY wORDS: Strategic analysis, strategy formulation, strategic decision.

\section{ANTECEDENTES}

Los sectores industriales, por lo general, se estructuran con pocas empresas que captan la mayor proporción del mercado y representan la parte sustantiva de la actividad económica y una mayor cantidad de medianas y pequeñas empresas que cubren áreas complementarias. En las empresas mayores, si la solución de los problemas estratégicos es deficiente, se constituyen cuellos de botella que impiden el desarrollo de las demás empresas $\mathrm{y}$ de otros sectores industriales.

Los temas empresariales, dentro del entorno de los negocios, pueden contemplarse desde dos enfoques contrapuestos: primero, el enfoque racional, que estima que los problemas son de tipo técnico y solamente se requiere la correcta aplicación de técnicas administrativas y de modernización tecnológica; segundo, el enfoque sistémico, que considera que existen diferentes concepciones y diferentes soluciones deseables, la solución requiere la discusión y la consideración de un rango de posibilidades factibles.

La falta de concertación y acuerdo es la causa frecuente de esa deficiencia decisional. Cuando las propuestas de solución o mejora se encuentran en conflicto, es necesario el diseño y empleo de herramientas que permitan la reducción de discusiones estériles, delimitando el rango de opciones de solución, factibles y deseables. La elección de adecuadas alternativas a los problemas de empresa deben considerar todas las opciones, basadas en información disponible y en el trazado de las probables consecuencias, dentro de una escala de preferencias orientada a lograr el máximo beneficio compartido de todos los grupos involucrados.

La sistematización de un grupo de herramientas para el análisis estratégico se enmarca dentro de un modelo de planeamiento estratégico. La elección varía en función a los cambios del entorno, las diferencias individuales, los intereses de grupo, los enfoques prevalecientes y los criterios personales de los actores.

1 Magíster en Administración, Ingeniero Industrial, Economista. Profesor en la Facultad de Ingeniería Industrial, Departamento de Producción y Gestión Industrial de la Universidad Nacional Mayor de San Marcos. E-mail: aacevedo@speedy.com.pe

2 Magíster en Dirección de Empresas por la Universidad de Piura, Ingeniero Industrial, estudios de Derecho. Profesora en la Facultad de Ingeniería de Sistemas e Informática de la Universidad Nacional Mayor de San Marcos. E-mail: klinares@speedy.com.pe

3 Magíster en Ingeniería Industrial, Ingeniero Industrial. Profesor en la Facultad de Ingeniería Industrial, Departamento de Producción y Gestión Industrial de la Universidad Nacional Mayor de San Marcos. E-mail: orestescachay@yahoo.es 


\section{EL MARCO CONCEPTUAL DEL ANÁLISIS ESTRATÉGICO}

El análisis estratégico permite mostrar si la empresa posee más debilidades que fortalezas o viceversa versus los competidores si el sector de negocio se vislumbra como atrayente, si la organización posee ventajas para competir, también relaciona el desempeño con las estrategias de crecimiento, estabilidad, contracción o combinada necesarias. Incluye la situación general de los productos definiendo cuatro tipos básicos: declinación, contracción u obsoletos, expansión o potenciales, en tránsito a la madurez y los maduros o principales.

\section{La percepción del problema de decisión estratégica}

La formación científica de los teóricos de la decisión explica la diversidad de soluciones en los problemas estratégicos, la falta de acuerdo en la confrontación de opciones y el conflicto ante la decisión tomada, ya que estos decisores poseen visiones cerradas y "duras" de los problemas, enfatizando la técnica y las soluciones óptimas y únicas (maximizadoras o minimizadoras).

Por otro lado, una metodología de evaluación cuantitativa con herramientas matriciales aumenta la posibilidad de consenso en la toma de decisiones directivas, ya que facilita la discusión y la unificación de criterios de decisión (al cuantificar la incidencia en los factores clave del desempeño y resultados). Conlleva la ventaja de enfocar la negociación en la mejor forma de ejecución de las acciones, porque disminuye el sesgo y subjetividad de los participantes.

\section{Las ventajas y desventajas del análisis estratégico}

En el análisis estratégico de una situación de insuficiente o inexistente análisis de largo plazo, se busca pasar a una situación donde se posea herramientas adecuadas para analizar y decidir cuáles serían las estrategias apropiadas, en función a los grandes fines de crecimiento y supervivencia de la empresa. Las herramientas matriciales son arreglos bifactoriales que facilitan la sistematización de los pasos secuenciales necesarios para encontrar las posibles estrategias, identificando su factibilidad y deseabilidad.

El empleo de matrices presenta las siguientes ventajas:
- Se puede evaluar secuencialmente a diversos conjuntos de estrategias.

- No existe un límite al número de estrategias que se pueden evaluar simultáneamente.

- Existe la facilidad de utilizar paquetes computarizados (p.e. hoja de cálculo).

- Se reduce sustantivamente el riesgo de no incluir factores clave.

- Se utiliza en una diversidad de organizaciones: públicas o privadas, internacionales o locales, grandes o pequeñas.

- Presenta un amplio espectro de uso, desde el análisis de sectores industriales, hasta el planeamiento de industrias y negocios.

Pero también presenta desventajas:

- Requiere pensamiento sistémico, visión estratégica, capacidad de aprendizaje continuo y habilidades para sintetizar y resumir datos frondosos en cuadros estratégicos.

- El decisor debe poseer experiencia debido a la probabilidad de sesgos por intereses y preferencias personales.

- Riesgo en la validez de los resultados por la frecuente inconsistencia de los datos de entrada.

- Posibilidad de conflicto en la fase de evaluación debido a diferentes conceptualizaciones.

\section{La eficacia del análisis estratégico}

El análisis por matrices es parte de un proceso de planeamiento de mayor nivel. Su resultado es la elaboración del fundamento para el diseño de las estrategias de producto específicas sobre las que se han de plasmar las estrategias de negocio, corporativas, funcionales y por unidad de negocio.

Su eficacia depende de fuerzas internas y externas, las que frecuentemente escapan al ámbito de control de los actores del planeamiento estratégico; por ello, es conveniente el empleo de las herramientas matriciales en forma selectiva y dosificada, considerando el aspecto específico que se analiza o se desea resolver dentro de organizaciones individuales enfocadas en segmentos de mercado, dentro de grupos empresariales que negocian en mercados ampliados o dentro de sectores industriales que se interrelacionan en mercados globales. 


\section{EL MODELO DE PLANEAMIENTO ESTRATÉGICO}

El planeamiento es uno de los principales sistemas directivos y se define como un sistema de actividad humana que proporciona elementos de juicio valederos para prever la visión de futuro, definir la misión interna, y evaluar el desempeño, a fin de establecer lineamientos que permitan la adaptación, supervivencia y crecimiento de la organización.

El planeamiento estratégico se define como un proceso analítico que facilita a la empresa la elección de cursos de acción, a través de identificar los componentes del sector, exponer los valores directivos, trazar las oportunidades-riesgos, visualizar las fortalezas-debilidades, definir la misión, especificar las estrategias factibles y deseables $(F+D)$, proponer alternativas de implantación, lo que permite la reducción de los riesgos y el aprovechamiento de las oportunidades del entorno.

\section{Definiciones estratégicas}

Las definiciones estratégicas básicas son:

- La Misión, que establece la razón de la existencia de la empresa y se operacionaliza con la definición del negocio.

- Los Objetivos, que presentan la posición que se espera alcanzar en un horizonte de planeamiento, reflejando las perspectivas, intereses y valores directivos vigentes.

- Las Metas, como objetivos operacionables y cuantificados.

- Las Estrategias, que son las líneas generales de acción.

- Las Políticas, son las guías de acción para la asignación de recursos.

- Los Planes, son los documentos explícitos del planeamiento, son corporativos, de negocio, funcionales y divisionales.

\section{Los subprocesos del planeamiento estratégico}

El Planeamiento Estratégico se plasma en un modelo, el cual consta -al primer nivel de resolución- de nueve actividades generales (véase Gráfico 1). Estas son:

Actividad 1: Concepción del sector industrial. Se refiere a qué es y cómo es el sector industrial donde se ubica la empresa, identifica las fuerzas intervinientes y la historia, tendencia y pronóstico. Se aplica un criterio general y globalizante.
Actividad 2: Concepción de la empresa. Se identifica la misión y los valores de la organización, conceptúa cuál es el negocio de la empresa a través de los valores directivos y las estrategias visibles.

Actividad 3: Análisis del conjunto estratégico externo. Diagnostica el conjunto estratégico externo y permite conocer los factores del entorno y su influencia en las áreas comercial y financiera, que son las primeras en sentir los efectos de los cambios y en amortiguar sus efectos al interior de la organización.

Actividad 4: Análisis del conjunto estratégico interno. Se analizan los aspectos de estructura, procesos y psicosociales, diagnostica los problemas internos, identifica las debilidades y define los procesos que generan valor agregado y diferenciación competitiva.

Actividad 5: Evaluación estratégica del desempeño. Analiza estática y dinámicamente (vertical y horizontal) los indicadores de resultados empresariales y evalúa el nivel de logro de la misión vigente en función a las variables de negocio, culmina en modificación o afirmación de la misión establecida.

Actividad 6: Análisis, síntesis y formulación estratégica. Aplica las herramientas matriciales para analizar y sintetizar las opciones factibles y calificar aquellas que se definen como deseables, determina las estrategias sobre las que se basa el diseño del plan.

Actividad 7: Especificación de estrategias F+D. En base a la posición estratégica de productosservicios, se diseña la estrategia general de desarrollo empresarial y se delinean las estrategias corporativas, funcionales, de negocio y de unidades estratégicas, llega a la elaboración del documento Plan Estratégico.

Actividad 8: Implantación del planeamiento operativo. Se refiere a los pasos necesarios para la adecuada aplicación del plan estratégico, incluye las decisiones de cambio y mejora, la metodología del modelo operativo de planeamiento, la gestión operativa, la estructuración del sistema de información estratégica para control de gestión.

Actividad 9: Seguimiento y evaluación estratégica. Contempla el control de avance de las variables de negocio e indicadores estratégicos de la empresa, determinando el nivel de logro y de resultados, se plasma en la reasignación de recursos para el logro de la misión. 
Gráfico 1. El proceso de planeamiento estratégicos.

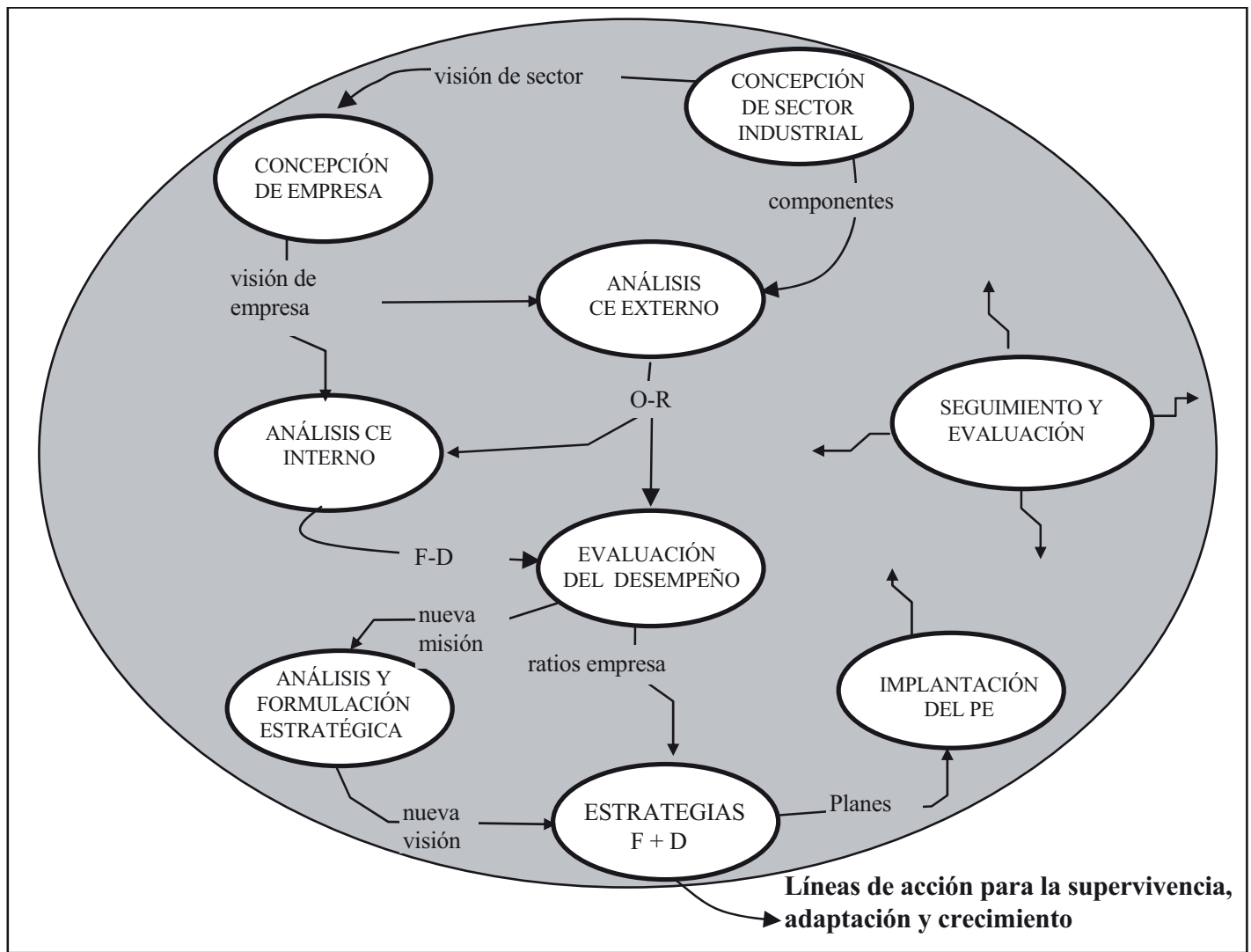

Fuente: Acevedo Borrego, Adolfo y Linares Barrantes, Carolina. El planeamiento estratégico en sectores regulados. Un caso práctico en telecomunicaciones. Revista AHCIET Nos 110, 111.

Cada actividad se considera un proceso transformador modularmente independiente, en el que el input es una visión parcial e incompleta del entorno y sus tendencias y el output sería una concepción gerencial más integral y profunda sobre el entorno y sobre el futuro de la empresa.

\section{EL SUBPROCESO DE ANÁLISIS Y FORMULA- CIÓN ESTRATÉGICA}

El análisis estratégico es la actividad del planeamiento que sintetiza el exhaustivo estudio realizado anteriormente. Esta actividad es un proceso de desagregación y síntesis por medio de arreglos gráficos multifactoriales, para la percepción de las estrategias adecuadas. Su elaboración sigue una secuencia lógica y constituye el enlace entre el análisis previo y la formulación posterior de los planes de largo plazo a partir de la misión y los grandes fines de crecimiento y supervivencia de la empresa. Permite la preparación de los objetivos globales, las estrategias generales, los objetivos funcionales y los planes y presupuestos detallados.

\section{Las fases del análisis estratégico}

Las cinco fases del análisis estratégico se muestran en el gráfico 2 .

La labor de análisis estratégico puede tomar semanas de trabajo laborioso y dedicado o sólo discusiones generales de una o dos horas, dependiendo del grado de conocimiento del sector, la experiencia en su manejo, la conceptualización del mismo y el grado de concertación que se logre en la discusión. Por esta razón se recomienda que los integrantes del equipo analista sean personas que se ubiquen en el nivel administrativo superior de las empresas y que posean pensamiento sistémico, una de las cinco disciplinas del aprendizaje en las organizaciones.

$1^{\circ}$ Fase de entrada, resume la información inicial, obtenida y organizada en las actividades previas. Considera tres asuntos relevantes: a) el medio ambiente, centrándose en las oportunidades y riesgos del ent-orno y el desempeño externo para determinar la posición estratégica externa; b) los sistemas empresariales, per- 
Gráfico 2. Análisis y formulación estratégica.

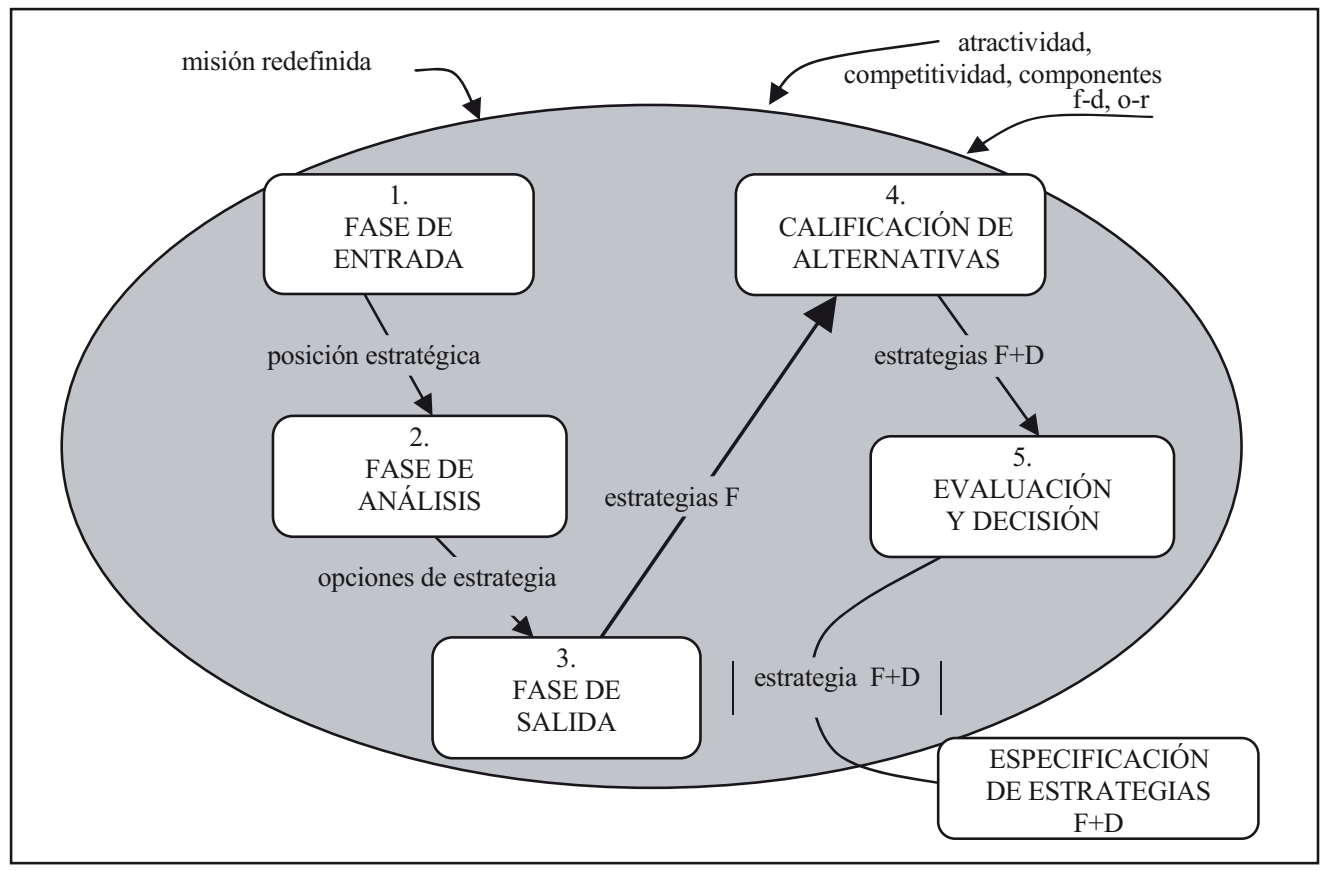

FuENTE: Acevedo, Adolfo (1993). Cuaderno de tareas en planeamiento estratégico. CENCAPH Serie Material Didáctico Hondutel, Honduras.

cibiendo las fortalezas y las debilidades que constituyen los factores internos clave sobre los que se establecen las estrategias y objetivos de las organizaciones; c) la estructura del sector que resume la información vital sobre los competidores, sus fortalezas y debilidades, las diferencias competitivas, el atractivo de la industria y las causas de éxito o fracaso en el sector. Esta fase plantea la posición estratégica de la empresa y los productos.

$2^{\circ}$ La fase de análisis, integra los factores externos e internos delimitados en la fase de entrada. Contempla tres puntos: a) el producto/mercado enfocado en los productos o servicios que ofrece el sector y los segmentos de mercado a los que se cubre; b) las divisiones o procesos de negocio centrales o descentralizadas (unidades de negocio desconcentradas o autónomas); c) el horizonte de previsión de largo plazo, para identificar probables eventos futuros y diversos escenarios. Esta fase plantea todas las alternativas estratégicas, independientemente de su viabilidad técnica.

$3^{\circ}$ La fase de salida, consolida todas las estrategias preparadas en la fase anterior a fin de verificar su factibilidad. Los criterios que permiten una adecuada preparación de las estrategias factibles son a) el marco estratégico como elemento deli- mitador de las fronteras, alcance y orientación de las opciones estratégicas (normatividad, valores gerenciales, misión y políticas); b) la priorización de problemas estratégicos en función a los escenarios previsibles, el atractivo de la industria y la asignación de prioridades financieras; c) el efecto del comportamiento de las variables del negocio en las estrategias generales (expansión, contracción, estabilidad, combinada), determinando las estrategias producto-mercado (negocio) y producto-funciones, d) el efecto de la posición y la coyuntura de la organización y del entorno en las estrategias generales (interna externa, relacionada no relacionada, activa pasiva, horizontal vertical).

$4^{\circ}$ La fase de calificación de alternativas, la valoración asignada a las diferentes opciones estratégicas está delimitada por su efecto en las variables de negocio (producto-mercado-funciones) o en los resultados empresariales (mejora de productividad, eficiencia, eficacia), de manera que se llegue a decisiones consensuadas. Mediante la metodología de valoración cuantitativa, se reduce la subjetividad y la confrontación de los participantes ya que se fijan las alternativas que cumplen dos condiciones: a) son factibles en el sentido que se pueden llevar a la práctica y b) son deseables en el sentido que no habrá fuerzas contrarias a su implementación, sean 
Gráfico3. Matrices de Análisis de problemas estratégicos.

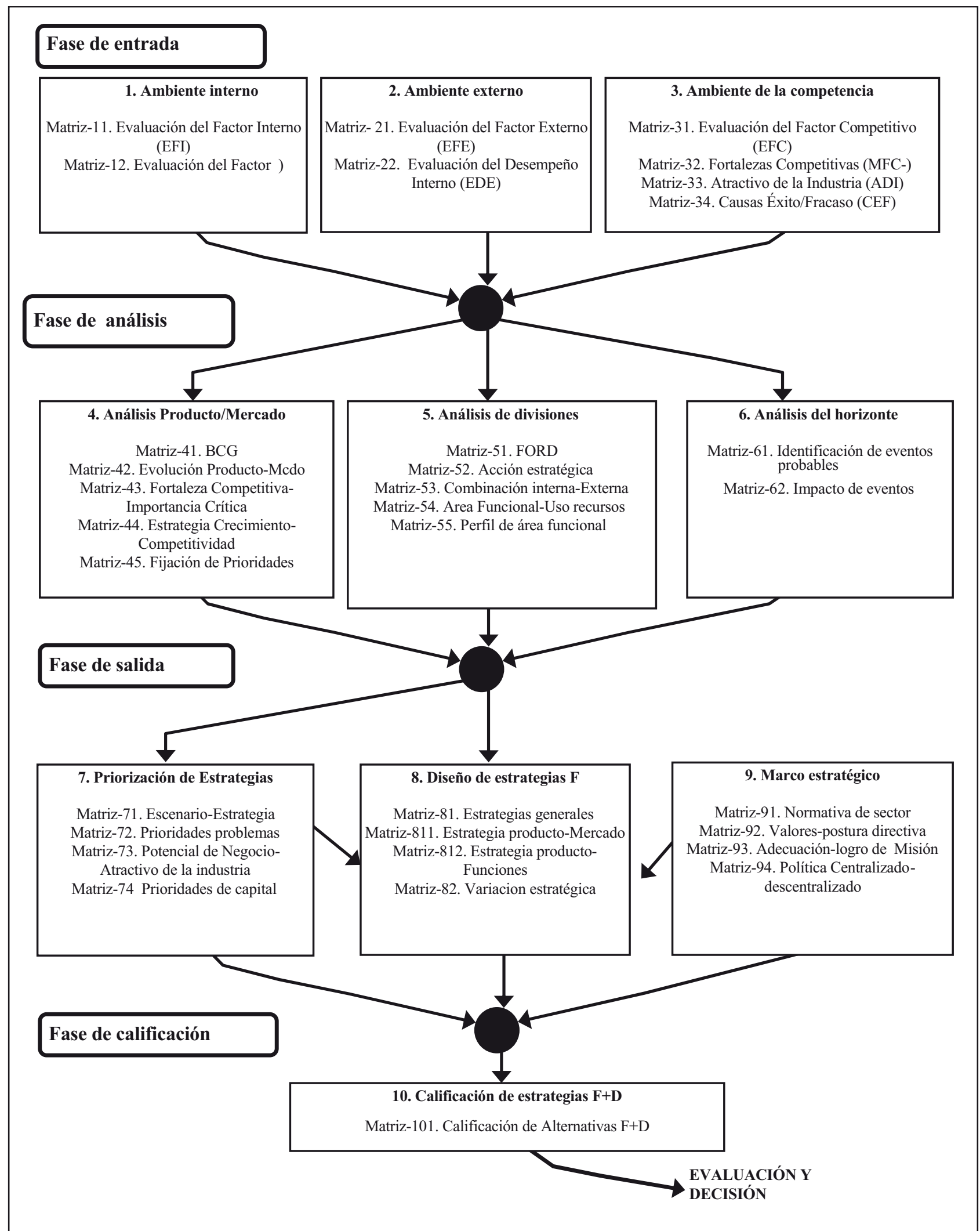

Fuente: Acevedo, Adolfo (1993). Cuaderno de tareas en planeamiento estratégico. CENCAPH Serie Material Didáctico Hondutel, Honduras. 
internas o del entorno. Se debe enfatizar que todas las opciones que cumplen la condición $F+D$ generan valor positivo, de manera que cualquiera sería aplicable a la empresa, en todo caso la calificación brinda elementos de juicio que facilitan la elección.

$5^{\circ}$ Evaluación y decisión, los términos de evaluación y conveniencia de las estrategias $F+D$ se discuten a la luz de los valores gerenciales y la misión enunciada por los decisores sectoriales o empresariales. El resultado más importante es la elección de estrategias específicas para la organización y la posibilidad de una adecuada negociación futura para la eficaz implementación, constituyendo los lineamientos que habrán de coadyuvar en el intento de disminuir la brecha entre lo real y lo deseado. Concretamente se prepara la formulación preliminar de las estrategias generales y de producto, sobre las que se han de especificar las estrategias de negocio, corporativas, funcionales y por cada unidad estratégica. Se requiere buen juicio y criterio y, en todo caso, no existe una respuesta única.

En el análisis estratégico se considera que de una situación inicial donde el análisis y la proyección de largo plazo son insuficientes o inexistentes, se pasa a una nueva situación con herramientas adecuadas para facilitar la elección y preparación de estrategias $\mathrm{F}+\mathrm{D}$.

\section{Descripción de las matrices}

Las actividades del análisis estratégico por matrices son: la fase de entrada que define la posición estratégica de la empresa y los productos, la fase de análisis que plantea todas las alternativas, la fase de salida que consolida las estrategias factibles, la fase de calificación que determina las estrategias $F+D$, la fase de decisión que elige la opción más apropiada (elevar el valor de la empresa) dentro del rango de alternativas $\mathrm{F}+\mathrm{D}$.

Para el desarrollo de estas fases se sigue una secuencia lógica para encontrar las estrategias viables, con herramientas gráficas multidimensionales (matrices). Comprende los criterios de decisión, la valoración de los factores clave, la cuantificación de su probable incidencia en el desempeño y la identificación de su factibilidad y deseabilidad (ver el gráfico 3).

\section{Pautas para la aplicación del análisis estratégico}

El planeamiento estratégico es un proceso que genera cambios en el ámbito personal: en visiones, percepciones y creencias sobre el sector, lo que lleva a nuevas actitudes, nuevo comportamiento y renovados resultados. Es un proceso de evaluación y preparación del diseño del futuro sectorial o empresarial, su objetivo es elaborar los elementos para la discusión y unificación de las diversas concepciones y weltanschauung's directivos.

La metodología de análisis estratégico posee ventajas: se puede evaluar simultánea o secuencialmente a diversos conjuntos de estrategias, no existe un límite al número de estrategias que se pueden evaluar simultáneamente, existe la facilidad de utilizar un paquete computarizado (Hoja de cálculo), se reduce sustantivamente el riesgo de olvidar alguno de los factores clave, es factible de utilizar en organizaciones, ya sean públicas o privadas, internacionales o locales, grandes o pequeñas empresas, es factible de utilizar para el análisis de sectores industriales y en la planificación del desarrollo sectorial e industrial.

\section{UN CASO EJEMPLO EN SECTOR DE SERVICIOS ALT-TEC}

El caso se enfoca en el sector de telecomunicaciones, donde la situación vigente de un operador incumbente al cual se controla para evitar posiciones de dominio y varios operadores de reducida inversión, es muy semejante en todos los países de la región.

\section{La necesidad del análisis estratégico para iden- tificar falencias sectoriales}

A partir de la privatización, se observó un salto en la oferta de telefonía fija, derivado de la liberalización de las tarifas reguladas de los servicios, un fuerte crecimiento en telefonía móvil, ingreso de nuevos actores orientados a servicios de avanzada tecnológica.

A pesar del gran desarrollo del sector que ha llevado a una demanda cubierta que equipara casi el $100 \%$ de la población total, se considera que la calidad de servicio no es la esperada y se acentúan las críticas al operador incumbente.

Un análisis estratégico, dentro de un proceso general de planeamiento, permite identificar las estrategias empleadas por los actores establecidos en 
el sector, lo cual representaría un importante elemento para plantear las acciones para fortalecer o cambiar la estrategia regulatoria empleada por cada operador del mercado, bajo la perspectivas de elevar su aporte para el total desarrollo de las telecomunicaciones.

\section{Sobre los hallazgos del análisis estratégico}

Telecomunicaciones es un sector integrador de otros sectores de diferente finalidad y diferentes modelos de negocio.

Por sus características de elevada inversión y la tendencia de su organización industrial hacia el monopolio natural, no es factible la existencia de un elevado número de operadores; empíricamente, se observa que el sector soporta un gran operador fijo de telefonía y banda ancha y otros operadores menores que se conectan a la red de la mayor empresa. En estas condiciones no existe la posibilidad de elevada competencia.

Aún así, las empresas diseñan su estrategia general de desarrollo buscando diferenciarse de las demás (ver Gráfico 4).

\section{Gráfico 4. La estrategia competitiva en el sector telecomunicaciones.}

\begin{tabular}{|l|c|c|}
\hline \multirow{2}{*}{} & \multicolumn{2}{|c|}{ Ventaja competitiva } \\
\cline { 2 - 3 } & Calidad & Costo bajo \\
\hline $\begin{array}{l}\text { Alcance } \\
\text { global }\end{array}$ & $\begin{array}{c}\text { Diferenciación } \\
\text { Cía.YY }\end{array}$ & $\begin{array}{c}\text { Liderazgo en costo } \\
\text { Cía. XX }\end{array}$ \\
\hline Segmentado & $\begin{array}{c}\text { Foco } \\
\text { Cía. ZZ }\end{array}$ & $\begin{array}{c}\text { Nicho } \\
\text { Cía. SS }\end{array}$ \\
\hline
\end{tabular}

FuENTE: Acevedo Borrego, Adolfo y Linares Barrantes, Carolina. El planeamiento estratégico en sectores regulados. Un caso práctico en telecomunicaciones. Revista AHCIET Nos 110, 111.

\section{Fuentes de competitividad sectorial}

A pesar de ser un sector no competitivo, telecomunicaciones requiere que los participantes sean actores competitivos. Cabe señalar que el concepto sector competitivo se refiere a la existencia de empresas (pocas o muchas) que se desenvuelven en un ambiente altamente dinámico, brindando valor diferenciado a los clientes, mediante el manejo de las variables de fuentes competitivas.
Se identifica que se aplican estrategias para desarrollar diversas fuentes de ventaja competitiva en las empresas, éstas son:

- Eficiencia y productividad en el uso de los recursos.

- Calidad y características de los servicios.

- Innovación en productos, servicios y procesos.

- Satisfacción y cumplimiento de las expectativas de los clientes.

Los operadores clásicos del sector han definido su propia estrategia competitiva y aplican estrategias diferentes para mantener un posicionamiento. La Cía. YY es el segundo actor del sector que busca diferenciarse por la calidad del servicio (cobertura y continuidad), la Cía. XX es un operador incumbente e integrador de diferentes sectores que aplica estrategia de bajo costo mediante estandarización de oferta y minimización de las funciones operativas, la Cía. ZZ considera que ha llegado a su tamaño óptimo de mercado y se dirige a clientes empresariales y nichos a los que brinda exclusividad al cubrir sus necesidades específicas.

El gráfico 5 muestra la estrategia competitiva y las fuentes de ventaja competitiva, aplicada por las empresas del sector.

\section{CONCLUSIONES}

El análisis y formulación estratégica, dentro de un modelo de planeamiento estratégico, integra la técnica y herramientas descriptivas que permiten la previsión y la identificación de elementos de juicio para la toma de decisiones directiva. Permite ejercitar el criterio y es importante para sistematizar las decisiones relevantes, para educar a los altos ejecutivos en la toma de decisiones de largo plazo, para mejorar la comunicación corporativa y para definir las consecuencias de la asignación de recursos.

El modelo general en sí es una herramienta para la toma de decisiones de la Alta Dirección que permite: conocer el atractivo del sector, identificar los factores clave internos y externos, evaluar la misión vigente y replantearla, diseñar estrategias factibles y deseables, brindar elementos para la formulación de los planes, brindar elementos para el control y la evaluación de los resultados de la gestión. 
Gráfico 5. Fuentes de ventaja competitiva en el sector.

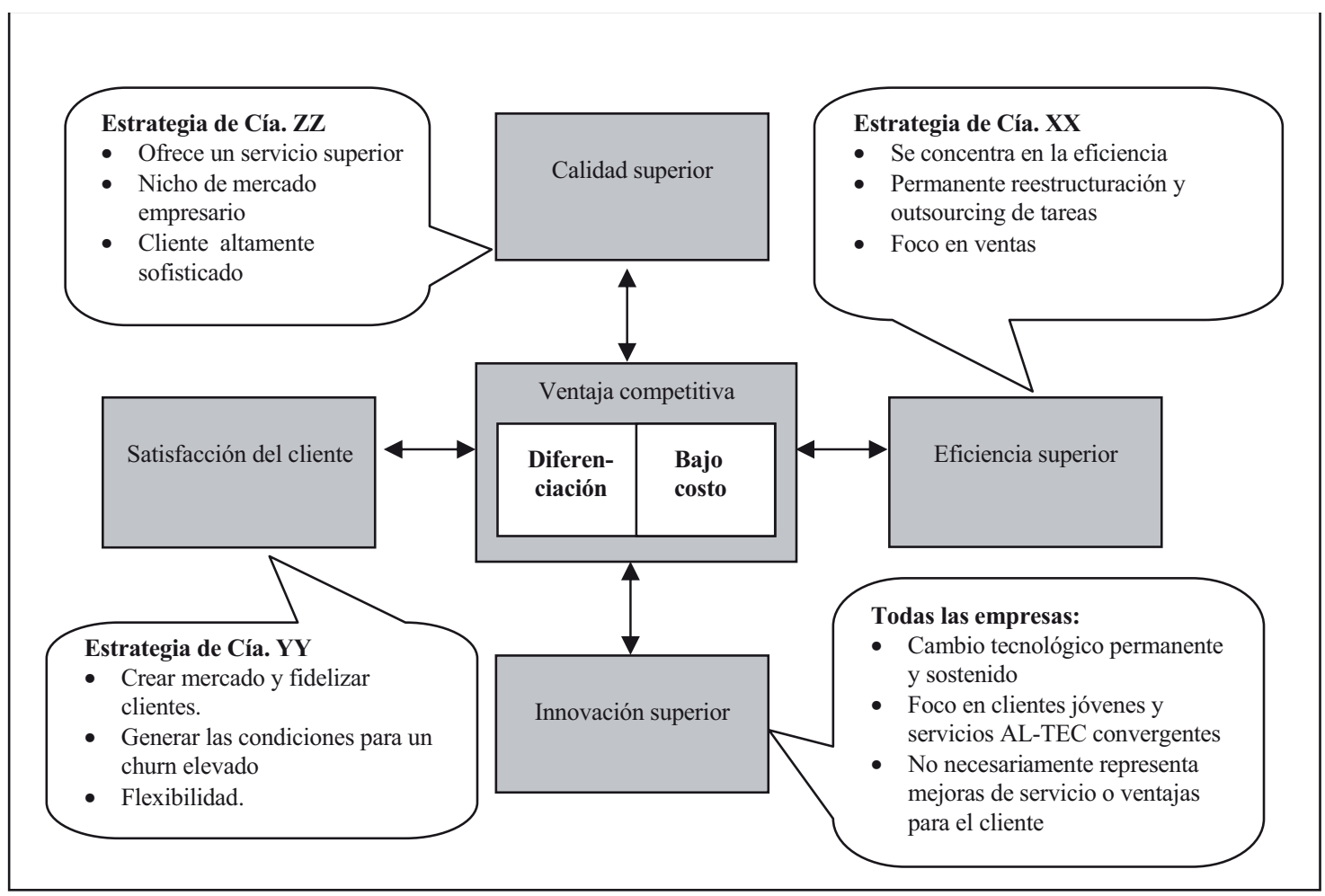

FuENTE: Acevedo Borrego, Adolfo y Linares Barrantes, Carolina. El planeamiento estratégico en sectores regulados. Un caso práctico en telecomunicaciones. Revista AHCIET N Nos 110, 111.

Dentro de las ventajas de su claridad y fluida aplicación, presenta diversas desventajas, ya que es necesario poseer juicio subjetivo y conjeturación educada, la validez de los resultados depende de la consistencia de los datos de entrada; son posible los sesgos y prejuicios debido a intereses, preferencias personales o conceptualizacion diferente.

\section{REFERENCIAS BIBLIOGRÁFICAS}

1. Acevedo Borrego, Adolfo y Linares Barrantes, Carolina. El planeamiento estratégico en sectores regulados. Un caso práctico en telecomunicaciones. Revista AHCIET Nos 110, 111.

2. Acevedo, Adolfo (1993). Cuaderno de tareas en planeamiento estratégico. CENCAPH Serie Material Didáctico Hondutel, Honduras.

3. Adell Hernani \& Martínez-Albelda (2002). La construcción de la Sociedad de la Información en España. Perspectiva 2001-2005. España.

4. Hax, Majluf (1994). Gestión de empresa con una visión estratégica. Ediciones Dolmen. Chile.

5. Hill, Gareth, Jones (1996). Administración Estratégica un enfoque integrado. Ed. McGraw-Hill/ Interamericana. México.
6. Linares Barrantes, Carolina (2005). Estudio descriptivo del segmento rural peruano: en el camino de crear la sociedad de la Información. Revista $A H C I E T$, №. 101. España.

7. Minztberg, Quinn y Voyer (1997). El proceso estratégico conceptos, contextos y casos. Ed. Prentice Hall. México.

8. Porter, Michael (1999). Ser competitivo nuevas aportaciones y conclusiones. Ediciones Deusto S.A. España.

9. Porter, Michael (1987). Ventaja competitiva creación y sostenimiento de un desempeño superior. Ed. CECSA. México.

10.Porter, Michael (1986). Estrategica competitiva técnicas para el análisis de los sectores industriales y de la competencia. Ed. CECSA. México.

11. Senge, Peter M (1990). La quinta disciplina. Editorial Granica S.A., México.

12.Stern, Deimler (2006). The Boston Consulting Group On Estrategy. John Wiley \& sons, Inc. USA.

13.Thompson y Strickland (1998). Dirección y administración estratégicas. Ed. Mc Graw Hill. México. 